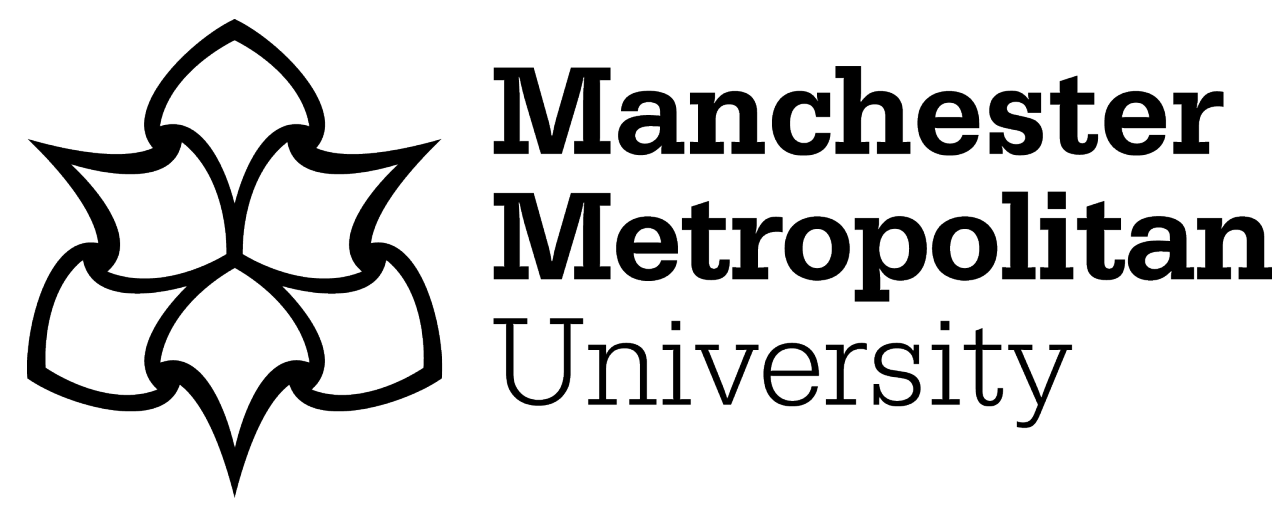

King, Laurie A ORCID logoORCID: https://orcid.org/0000-0002-0772-2378 and Parkinson, BA (2017) Probing the Relative Photoinjection Yields of Monomer and Aggregated Dyes into ZnO Crystals. Langmuir: the ACS journal of surfaces and colloids, 33 (2). pp. 468-474. ISSN 0743-7463

Downloaded from: https://e-space.mmu.ac.uk/624302/

Version: Accepted Version

Publisher: American Chemical Society (ACS)

DOI: https://doi.org/10.1021/acs.langmuir.6b03395

Please cite the published version 


\title{
Probing the Relative Photoinjection Yields of Monomer and Aggregated Dyes into Zno Crystals
}

\author{
Laurie A. King ${ }^{1}$ and B. A. Parkinson ${ }^{1}$ \\ ${ }^{1}$ Department of Chemistry and School of Energy Resources \\ University of Wyoming \\ 1000 E University \\ Laramie, WY 82071
}

\section{TOC Image}

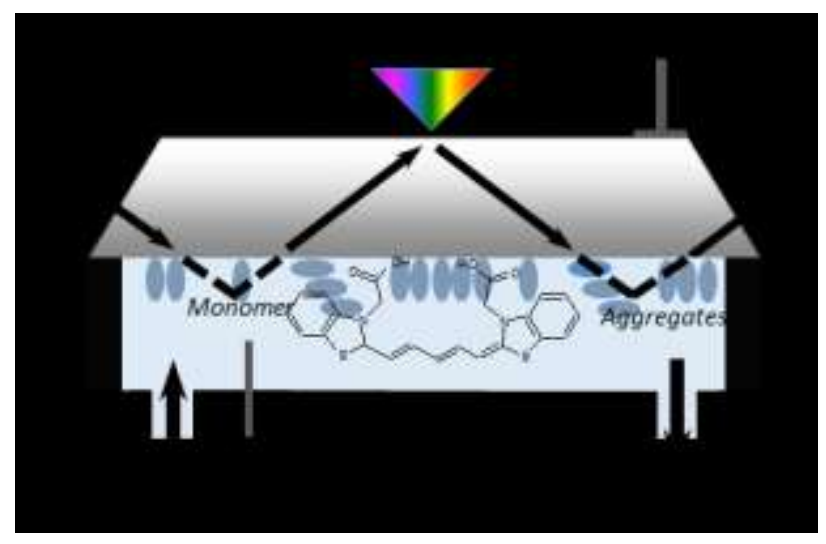

\begin{abstract}
Cyanine dyes, often utilized in dye-sensitized solar cells (DSSC), form a range of molecular species from monomers to large $\mathrm{H}$ - and J- aggregates in both solution and when adsorbed at a photoelectrode surface. To determine the relative capability of the different dye species to inject photo-excited electrons into a wideband gap oxide semiconductor, sensitization at a single crystal zinc oxide surface was studied by simultaneous attenuated reflection (ATR) UV-vis absorption and photocurrent spectroscopy measurements. ATR measurements enable identification of the dye species populating the surface with simultaneous photocurrent spectroscopy to identify the contribution of the various dye forms to photocurrent signal. We study the dye 2,2' carboxymethylthiodicarbocyanine bromide that is particularly prone to aggregation in both solution and at the surface of sensitized oxide semiconductors.
\end{abstract}




\section{Introduction}

Dye-sensitized solar cells (DSSCs) are an inexpensive and readily scalable solar cell technology that have attracted significant attention from the scientific community.$^{1-4}$ DSSCs are comprised of a sensitizer, a large band gap semiconducting material, a charge transport redox electrolyte and counter electrode. In conventional DSSCs upon absorption of a photon, an electron is promoted from the HOMO to LUMO level of the dye with subsequent separation of the photogenerated electron-hole pair by injection of the electron into the conduction band of the semiconductor. Typically the semiconductor is mesoporous titanium dioxide $\left(\mathrm{TiO}_{2}\right)$ but in some cases high surface area zinc oxide $(\mathrm{ZnO})^{5-7}$ or other oxides such as tin oxide $\left(\mathrm{SnO}_{2}\right)^{8,9}$ are used. Charge collection and regeneration of the surface bound oxidized dye occurs by oxidation of redox species in the electrolyte in competition with recombination pathways via the oxidised redox species.

Ruthenium complex dyes, in particular the complex cis-di(thiocyanato)-bis(2,2'bipyridyl-4,4'-dicarboxylate) ruthenium (II) (commonly called N3) and other related ruthenium based dyes, remain the most commonly utilized sensitizers in DSSCs due to their stability and broad spectral absorbance range. ${ }^{10-12}$ However, organic dyes such as cyanine dyes, are attractive alternatives for DSSC technologies due to their synthesis from abundant elements and high absorptivities. ${ }^{13,14}$ Cyanine dyes are prone to aggregation, forming a range of molecular species in both solution, ${ }^{15}$ and when adsorbed on a photoelectrode surface. ${ }^{16-18}$ Such aggregates form with different geometric arrangements of dye monomers leading to distinct blue or red shifts and are labelled as $\mathrm{H}$ - or J- aggregates, ${ }^{19}$ where $\mathrm{H}$ aggregates have blue shifted and J aggregates have red shifted absorbance relative to the dye monomer.

Historically the fundamental science that underpins DSSCs began with the sensitization of silver halides to visible light for application in photography. However, it was during the 1960s when the sensitization of single crystal metal oxide substrates with non-covalently bound dyes was motivated for capturing solar energy. ${ }^{20-23}$ Further innovation led to the utilization of covalently bound dyes, that enhanced electronic coupling of the dye to the surface. ${ }^{24,23}$ In the 1990's dye sensitization received renewed attention due to the utilization of covalently bound dyes, in 
combination with mesoporous titanium dioxide that increased the metal oxide surface area by 1000 fold leading to significantly higher dye coverage and hence enhanced light absorption and resulting solar cell efficiencies. Despite the practical benefits of utilizing mesoporous substrates, the porous structure adds a degree of complexity. Therefore there remains a compelling advantage to using well characterized single crystal substrates to uncover the fundamental scientific underpinnings of DSSCs. For example, investigations into the dependence of photocurrent and photovoltage on the doping density of single crystal $\mathrm{TiO}_{2}$ substrates. ${ }^{27}$ Other studies have probed the influence of "lattice matching" of various surface-dye combination ${ }^{28-30}$ where the highest dye loading and photocurrent yields were recorded for samples where the distance between the two binding carboxylate groups of the dye molecule most closely matched the Ti binding sites on the single crystal surface.

Given the inherently low optical density of a monolayer or submonolayers of dye adsorbed to a single crystal surface, specialized techniques are needed to probe surface bound dye sensitization such as photochronocoulometry, ${ }^{31}$ and attenuated total reflection spectroscopy (ATR) that provides spectral information about surface adsorbed dye species. To perform ATR spectroscopy, a single crystal is utilized as both a working electrode and attenuated total reflection element (ATRE) ${ }^{32-35}$ to simultaneously acquire ATR and photocurrent incident-photon-conversionefficiency (IPCE) spectra enabling the correlation between surface bound dye populations and their effectiveness as sensitizers.

ATR has been used to study both non-covalently bound ${ }^{34,36}$ and covalently bound ${ }^{35}$ sensitizing dyes. Given the distinct optical shift from monomer to aggregate for cyanine dyes, ATR is an essential tool to decipher surface bound populations and their contribution to photocurrent yields. Matches ${ }^{35,37}$ and disparities ${ }^{34,36,37}$ between the spectral shapes of absorbance and photocurrent yields have been observed for different sensitizers. In this paper we probe the attachment and sensitizing photocurrent from monomer and aggregated 2,2' carboxymethylthiodicarbocyanine bromide (given the abbreviation R8) dye at a $\mathrm{ZnO}$ single crystal surface by simultaneous ATR and photocurrent measurements. R8 has a nearly identical 
molecular structure to G15 (2,2' dimethylcarboxy-thiacarbocyanine iodide) that we have previously studied with ATR techniques. ${ }^{32}$ The structural difference is an additional 2 carbons in bridging carbon chain between the two cyanine groups extending the conjugation length from 3 to 5 carbons as shown in Fig. 1. R8 has a red shifted absorption maximum and is known to aggregate much more readily than $\mathrm{G} 15,30$ allowing further investigation of aggregation effects on photoinjection yields.

A.

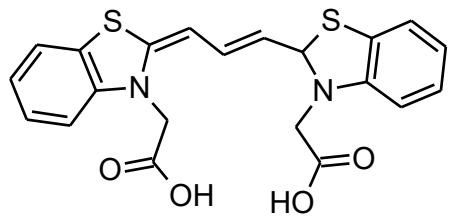

B.

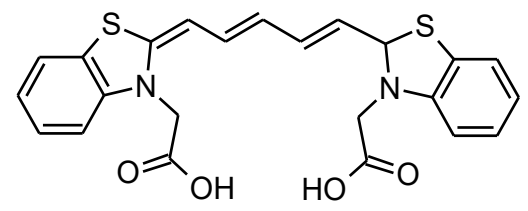

Figure 1. Molecular structures of (A) G15 and (B) R8 dyes.

\section{Experimental}

\section{Zinc Oxide-crystal ATRE}

A ZnO (0001) (MTI corporation) $10 \times 10 \times 1 \mathrm{~mm}$ crystal with a doping density of $3.00 \times 10^{17} \mathrm{~cm}^{-3}$ and was fashioned into a prism shaped ATRE by polishing the two 1 $\mathrm{x} 10 \mathrm{~mm}$ opposite faces of the crystal at a 30-degree angle relative to the normal such that the longest face was the zinc terminated face. Both faces of the ATRE were polished with $0.04 \mu \mathrm{m}$ followed by $0.02 \mu \mathrm{m}$ silica solution (MasterMet ${ }^{\mathrm{TM}} 2$ Non Crystallizing Colloidal Silica Polishing Suspension, Buehler) on a polishing pad and subsequently annealed at $1000{ }^{\circ} \mathrm{C}$ in air for $2 \mathrm{~h}$. All experiments were performed on the $<0001>$, zinc face, of the crystal. The oxygen face is readily identified by the formation of etch pits leading to a rapid white discoloration upon contact with dilute acid. A terraced, atomically flat surface was confirmed by atomic force microscopy (AFM) (Asylum Research Cypher) using K-TEK Nanotechnology NSG30 tips prior to sensitization experiments (Figure 2.B).

\section{Dicarboxylate Cyanine Dye}

The dicarboxylate cyanine dye, R8 (2,2' carboxymethylthiodicarbocyanine bromide) was synthesized following Ref ${ }^{38}$. Several different aqueous $\mathrm{R} 8$ dye solution 
concentrations were prepared $\left(1.08 \times 10^{-6} \mathrm{M}-67.5 \times 10^{-6} \mathrm{M}\right)$ by serial dilution for ATR measurements.

\section{UV-vis Solution Absorption Spectra}

All solution absorption spectra were obtained using a Perkin-Elmer Lambda 950 spectrophotometer with either $2 \mathrm{~mm}$ or $10 \mathrm{~mm}$ path length cuvettes.

\section{Simultaneous IPCE and ATR data acquisition}

The ZnO crystal was utilized as both a working electrode (for photocurrent spectroscopy measurements) and an ATRE for optical measurements. The in-house designed Teflon ${ }^{\circledR}$ flow cell was used for ATR and photocurrent measurements was described elsewhere. ${ }^{35}$ As depicted in Fig. 2A, the zinc oxide crystal was held in position in the flow cell, facilitating the injection/removal of dye and electrolyte solutions. Atomic force microscopy was used to confirm a clean, atomically flat, terraced ZnO surface prior to ATR measurements (Fig. 2B).

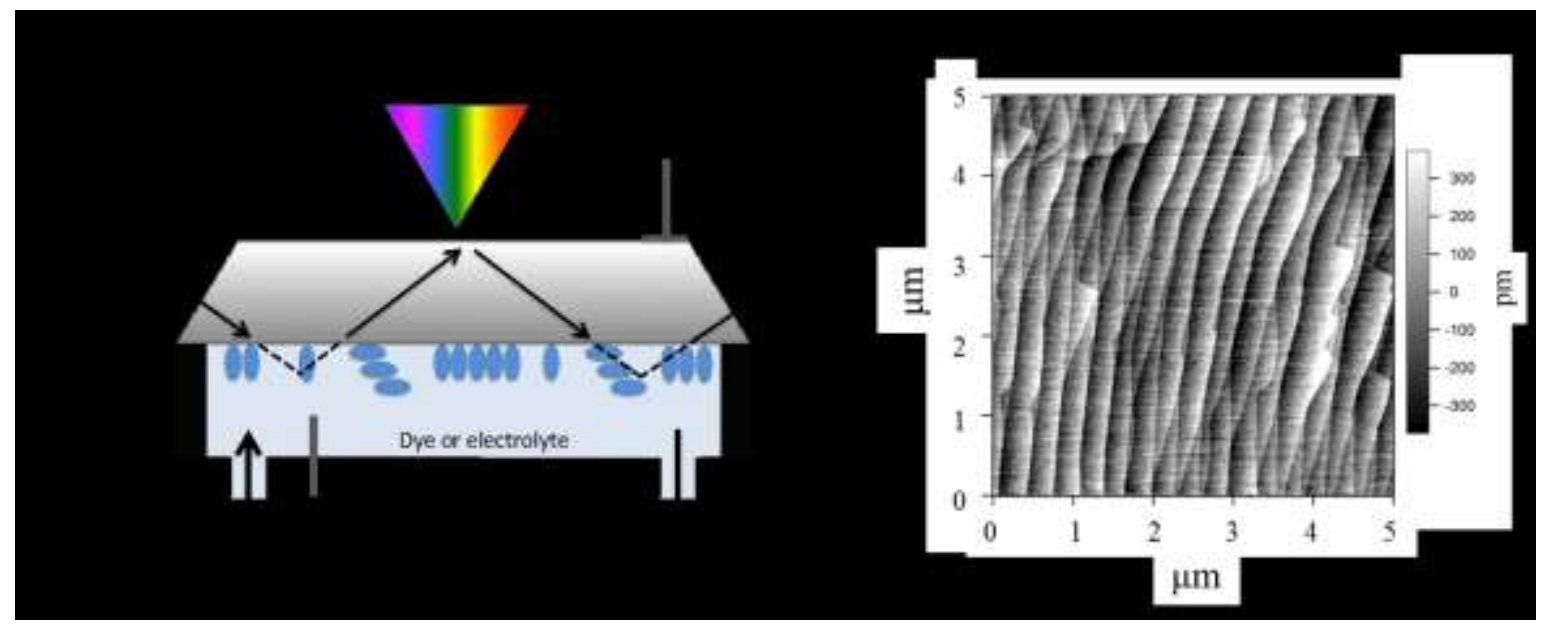

Figure 2. (A) Schematic of the experimental setup for simultaneous acquisition of photocurrent and ATR spectra. Monochromatic light is used as the photocurrent probe (back side illumination). A white light probe delivered to the crystal at $30^{\circ}$ relative to the long side of the ATRE is used for ATR spectroscopy. (B) AFM micrograph of a clean, terraced ZnO ATRE (0001), zinc face.

A halogen lamp (Newport) in conjunction with a motorized monochromator (Jarrell-Ash Fischer Scientific) was used to produce a monochromatic light beam for photocurrent spectroscopy. The monochromatic light passed through a $400 \mathrm{~nm}$ long 
pass filter and was focused with a focusing lens onto the long side of the zinc oxide ATRE (back side illumination). A potentiostat (Princeton Applied Research, model 174) and lock-in amplifier (Stanford Research, model SR830) were used with an inhouse developed program to collect the photocurrent data. Two electrode measurements were conducted with a platinum wire as a counter electrode. Spectra were collected between 800 and $400 \mathrm{~nm}$ with a $2 \mathrm{~nm}$ step-size. $10 \mathrm{mM}$ hydroquinone (a fast-redox couple) as regenerator with $50 \mathrm{mM}$ tetrabutylammonium hexafluorophosphate as electrolyte (Fluka, electrochemical grade) in acetonitrile (Fischer Scientific, HPLC grade) was used for all of the photocurrent measurements. All measurements were conducted at short circuit potential.

White light (Hamamatsu, high power UV-vis fiber light source) was coupled into the ATRE with a single optical fibre (Oz Optics LTD) and focused with a focusing lens onto the $\mathrm{ZnO}$ ATRE at a 30 degrees angle relative to $\mathrm{ZnO}$ front face. Light transmitted through the crystal was collected with a second focusing lens and directed onto an optical fibre bundle (Fiberguide Industries) that led directly into a CCD detector (CBEx 980 with special temperature stabilization, Snowy Range Instruments). The software (Snowy Range Instruments) was used to collect and average data. 600 spectra were collected and averaged for one data set. Prior to dye sensitization, a background measurement was obtained with the unsensitized $\mathrm{ZnO}$ crystal with the cell full of solvent and used as the reference data to calculate absorbance data. The penetration depth of the incident white light probe was calculated to be at least 80 $n m$ into the electrolyte species - beyond the surface of the ZnO ATRE surface.

The $\mathrm{ZnO}$ single crystal was sensitized by sequentially injecting low to high concentrations of dye solutions (in $18.2 \mathrm{M} \Omega$-cm water) into the ATR flow cell. Each solution concentration remained inside the flow cell for $30 \mathrm{~min} .{ }^{28,30}$ The dye solution was then removed by flushing the cell with $5 \mathrm{~mL}$ of water followed by $15 \mathrm{~mL}$ of acetonitrile. Subsequently, electrolyte solution was pushed into the cell and the system was probed with photocurrent and ATR absorption spectroscopies. Prior to further sensitization, the cell was flushed with $15 \mathrm{~mL}$ of water. This cycle was repeated for each of the dye concentrations studied.

\section{Determination of monomer and dimer spectra}


A known molecular concentration of aqueous R8 dye was prepared as a stock solution $(77.0 \mu \mathrm{M})$. A serial dilution was performed from the stock solution to prepare a total of 5 dye concentrations (37.3, 1.50, 0.75 and $0.30 \mu \mathrm{M})$. The distinct monomer and dimer spectra were determined using the basic assumption that two monomers are in equilibrium with a dimer:

monomer + monomer $\Leftrightarrow$ dimer

(Equation. 1)

A multi-variate program, SPECFIT/32 (Spectrum Software Associates, Chapel Hill, NC) was used to deconvolute overlapping absorbances to determine the pure monomer and dimer spectra with an unknown equilibrium constant, K. The whole set of spectra were globally analyzed and fit to a dimerization equilibrium model (Equation 1) employing factor analysis by Singular Value Decomposition (SVD) and non-linear least squares fitting to obtain a dimerization constant, $\log (\mathrm{K})=3.536$ as well as the monomer and dimer absorptivity spectra and therefore extract the monomer and dimer concentrations in a given solution.

\section{Results and Discussion}

The absorption spectra of a series of R8 dye solutions are shown in Fig. 3a. The absorbance data for a set of dye solutions were normalized with respect to their total molecular concentrations ( $\mathrm{C}_{\text {tot }}$ ). As shown in Fig. 3b, there are two isobestic points in the absorptivity data at $610 \mathrm{~nm}$ and $680 \mathrm{~nm}$. The isobestic point at $680 \mathrm{~nm}$ is red shifted with respect to the monomer and is therefore assigned to the presence of a very low concentration of J-aggregated dimers. Characteristic cyanine J-aggregate spectra are narrow, and intense in comparison to $\mathrm{H}$-aggregate peaks. ${ }^{14}$ Therefore this rather broad, feature indicates this dimeric structure is rather weakly bound and differs from a typical J-aggregate. ${ }^{39}$ The rather distinct blue shifted isobestic point is assigned to $\mathrm{H}$-aggregation, mostly dimerization, of monomer since further aggregation would not produce such a distinct isobestic point. 


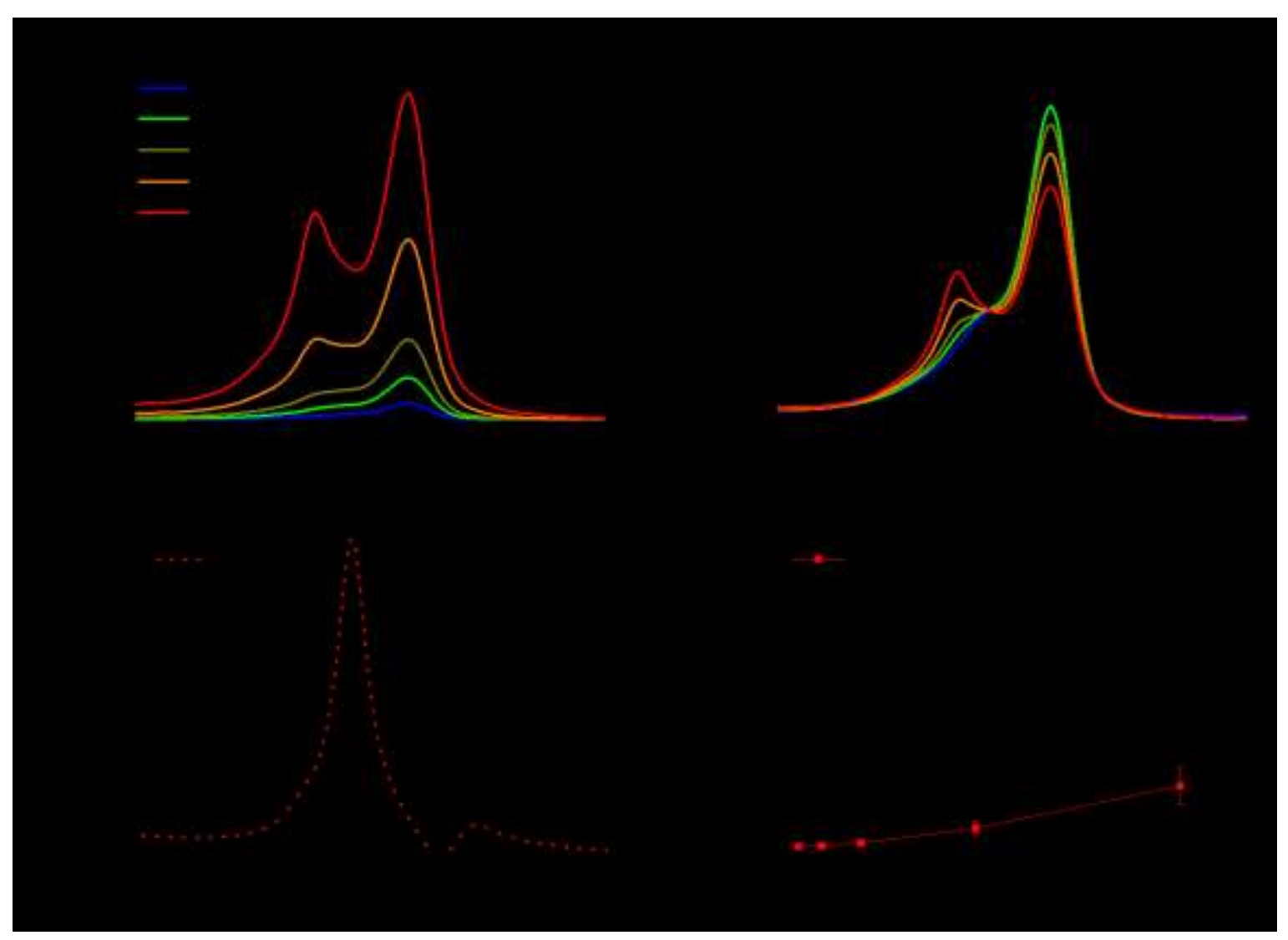

Figure 3. (a) UV-vis solution absorption spectra and (b) absorptivity spectra used to determine the monomer and dimer spectra. (c) Absorptivity plots of monomer (black, continuous line) and dimer (red, dashed line). (d) Monomer (black, squares) and dimer (red, circles) concentration profiles plotted as a function of total solution concentration.

The deconvoluted monomer and dimer spectra are shown in Fig. 3c. Given the small magnitude of the J-aggregation $(\sim 710 \mathrm{~nm})$ the fit was forced for just two species, monomer and H-aggregate dimer; hence the presence of an additional peak in the dimer spectra at $\sim 700 \mathrm{~nm}$. The absorption maximum of the monomer spectra is approximately $660 \mathrm{~nm}$ with the dimer at $586 \mathrm{~nm}$.

Simultaneous ATR and photocurrent (IPCE) measurements were performed over two concentration ranges of sensitizing dye and are shown in Fig. 4 (two separate experiments). Overall, the general shape of the IPCE data matches that of the R8 solution absorption data indicating that both the monomer and aggregated dye species are both able to photoinject and contribute to IPCE signal. Across each dataset, the magnitude of ATR optical absorbance and IPCE both increase with sensitizing dye concentration implying an increase in the total number of adsorbed 
sensitizing dye molecules. Since the carboxylate groups binding the dye to the surface are labile enough to permit "walking" via one bond being dissociated, but not so labile that both dissociate at once resulting in desorption, ${ }^{35}$ as the dye concentration increases, the dye becomes packed closely on the surface and will start to reorganize to accommodate more adsorbed dye molecules and interact to form aggregates that may vary in structure due to the geometry of the surface binding sites.

It is striking that the IPCE maxima are approximately equal in magnitude $(\sim 3.5 \mathrm{x}$ $10^{-4}$ ) for the two experiments, albeit with different spectral shapes (different concentrations of monomer and dimer adsorbed species). These values are approximately one order of magnitude lower than maximum IPCE values $(3 \pm 0.5 \mathrm{x}$ $10^{-3}$ ) measured for G15 sensitization on $\mathrm{TiO}_{2}$ crystals with an optimized doping density in the range of $10^{17} \mathrm{~cm}^{-3}$. These values are close to $100 \%$ absorbed photon current efficiency (APCE) where every absorbed photon results in a photocurrent signal. IPCE values with non-optimum higher or lower doping densities were closer to those measured in these experiments suggesting that recombination between injected electrons and oxidized dye is limiting the photocurrent in these experiments. Given that the total time for dye adsorption is equal ( 5 dye concentrations, each sensitized for $30 \mathrm{~min}$ ) in the two experiments, we speculate that the difference in IPCE spectral shapes is due to differing kinetics of dye adsorption (and desorption) from the monomer and aggregates in solution, thus leading to a different proportion of adsorbed dye species, but a similar total number of dye molecules sensitized. Regarding the similar maxima in the IPCE for the two different experiments (Figure $4 \mathrm{~A}$ and $\mathrm{B}$ ), we speculate that a difference in dye concentrations leads to a different packing and organisation of dye species at the surface, and perhaps beyond, into solution. Indeed, at the higher concentration range, there is a higher proportion of aggregate. At the lower concentration range, monomers dominate the solution and surface. At the higher concentrations, we speculate that fast adsorption leads to a less ordered surface with, perhaps, less efficient packing of dyes. 


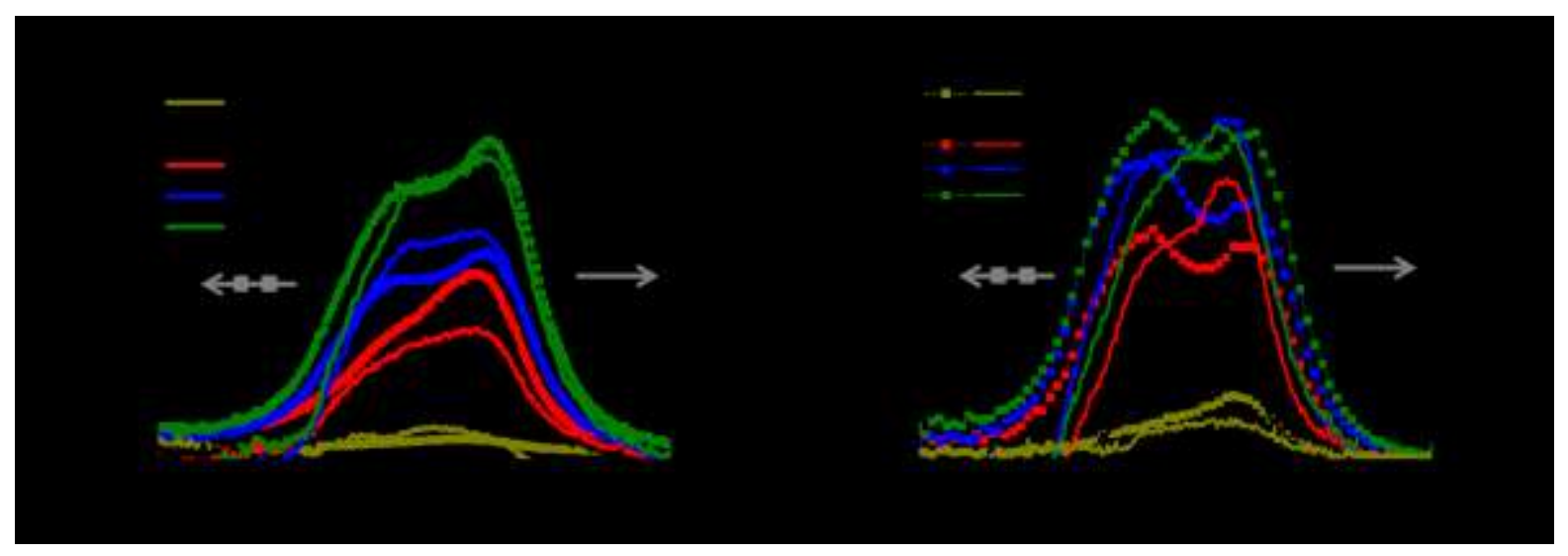

Figure 4. Simultaneously collected photocurrent and ATR absorbance spectra for $\mathrm{ZnO}$ sensitized with (a) $1-7.5 \mu \mathrm{M}$ and (b) $1.5-67.5 \mu \mathrm{M}$ R8 dye (aqueous). These data were collected in two separate experiments each starting with the same terraced, unsensitized $\mathrm{ZnO}$ single crystal surface.

Given the spectral broadening of the surface spectra (compare Figures 3 and 4), to further evaluate the monomer and aggregate contributions to the IPCE spectra the IPCE to absorbance ratios are plotted as a function of concentration in Fig. 5. Specifically, we plot ratios of $670 \mathrm{~nm}$ to $610 \mathrm{~nm}$ wavelengths that represent the proportion of monomer to aggregate. Over the lower concentration range probed in these experiments, the IPCE and absorbance data appear to track each other with no large discrepancy between absorbance and IPCE values. Thus, we conclude that across the low concentration range photocurrent is equally derived from both monomer and aggregated species and is in proportion to their surface concentration as was the case with the previously studied G15 dye. However at the higher concentration range (16.3 - 67.5 $\mu \mathrm{M}$, Fig. 4B) there is some deviation between the IPCE and absorbance spectral shapes indicating that the H-aggregate is more efficient at producing photocurrent than the monomer. 


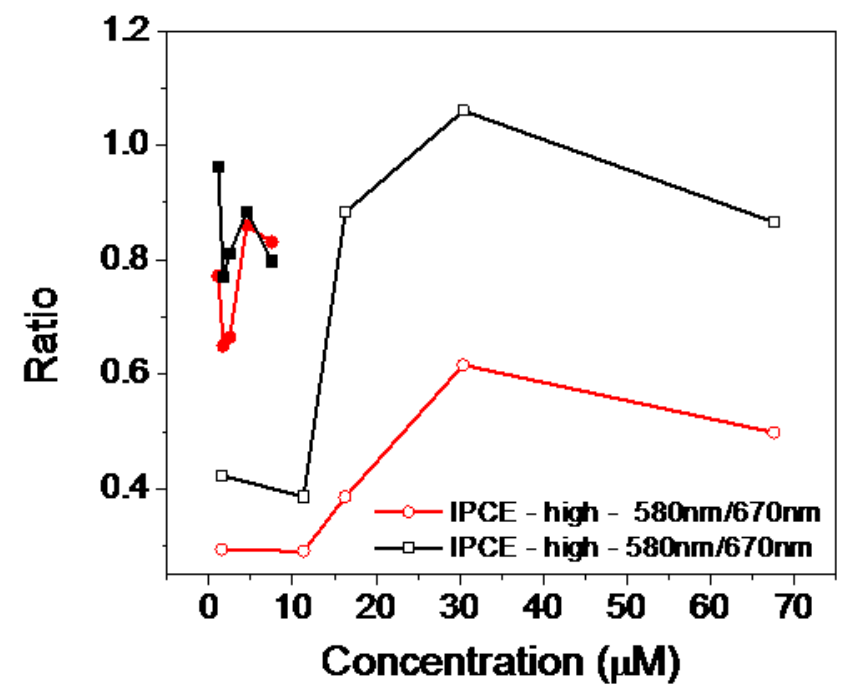

Figure 5. Ratio of dimer $(580 \mathrm{~nm})$ to monomer $(670 \mathrm{~nm})$ for both IPCE (black) and absorbance data (red). The data is plotted as a function of the dying concentration and is taken from Figure 4. The lower concentration range (Figure 4.A) is shown with filled symbols; the higher concentration range (Figure 4.B) is plotted with open symbols.

\section{Discussion}

The IPCE for dye sensitization depends on several factors. First, the amount of light absorbed by the adsorbed dye producing the excited state that in our case is a small fraction of the incoming light due to the thin layer of adsorbed dye. The ATR measurement directly gives us the relative amounts of light absorption. Secondly, the injection efficiency of the photexcited electron into the semiconductor substrate due to the competition between the relaxation rate of the excited state and the injection rate into the semiconductor conduction band. In the case of strongly covalently attached dyes the rate of injection is in the femtosecond regime while the dye excited states last picoseconds or more meaning the injection efficiencies are near unity. Finally, the recombination rate of the injected carrier, either in the semiconductor or by a return of the photoinjected electron to an adsorbed photooxidized dye, can reduce the IPCE values.

To understand why the $\mathrm{H}$-aggregate might be more efficient at photocurrent generation (IPCE) than the monomer several possible mechanism will be discussed below. We first review our previous results where the sensitization of $\mathrm{ZnO}$ with 2,2' dimethylcarboxy-thiacarbocyanine iodide (G15, Fig. 1B) dye was determined by 
simultaneous ATR and photocurrent spectroscopies. ${ }^{35}$ In this study agreement between the optical absorbance and photocurrent spectra for G15 was observed over the entire range of surface coverages. It was concluded that the electron injection and collection yields are approximately the same regardless of whether the dye was in the form of a monomer or an aggregate (dimer). Whereas matching ATR and photocurrent spectra are relatively easy to interpret (both monomer and aggregated species contribute equally to their surface population), disparities between the two measurements requires further interpretation. Both dye and sensitization literature provides possible explanations for such disparities, which are briefly discussed here. (Doping level discrepancy discussed here if it is applicable).

Firstly, a difference in oxidation potentials of excited/ground state monomer and aggregated species may lead to a difference in driving force for electron injection due to the higher energy excited states of H-aggregates. ${ }^{40,17}$ Alternatively, this difference could influence the strength of the electronic coupling between the excited monomer/aggregate and $\mathrm{TiO}_{2}$ acceptor states. ${ }^{17}$

A hole-trapping mechanism is an alternative explanation for differing photocurrent yields from monomer and dimer/aggregate species. Initially proposed in the context of the photographic process, ${ }^{37}$ the hole-trapping mechanism considers that dye aggregates can contain trap states for excitons. In this model, the exciton of an aggregated species is dissociated by trapping of the hole at either surface defect states, or, due to adjacent monomer/aggregate species giving rise to differences in the monomer/aggregate contribution to photocurrent efficiencies relative to the surface adsorbed population. This mechanism would decrease the injection yields from large aggregates.

Studies directly measuring recombination rates by transient absorption techniques have been performed with many different dyes such as zinc phthalocyanine, ${ }^{41}$ squaraines ${ }^{42}$ chalcogenorhodamines, ${ }^{18}$ amongst others ${ }^{43}$. In each of these studies, injection and/or recombination kinetic pathways of dye excitons were found to change as a function of aggregation. Such discrepancies are often described as changes in the dye adsorption geometry (which differs with respect to 
aggregation) and therefore accredited to differences in the tunnelling barrier height for injection/recombination.

One further explanation for discrepancies between monomer and aggregate injection efficiencies reported in the literature is based on the fact that a wide band gap semiconductor surface may have so called "hot spots" for injecting electrons. ${ }^{44} \mathrm{~A}$ monolayer of sensitizing dye can show a difference between monomer/aggregate injection yields arising from efficient exciton migration through aggregates compared to hopping between monomeric species. The enhanced migration thus increases the probability that excited electrons are injected into the semiconductor "hot spots".17,44

To evaluate which of the fore mentioned processes may be operating in our case the energetics of G15 and R8 ground and excited states relative to $\mathrm{ZnO}$ bands is determined. Using literature values for the oxidation potential of the dyes, the excited state energies of the monomers can be calculated if the excitation (optical measurement) and reorganization energies are also known. The reorganization energy for these dyes is known to be about $0.3 \mathrm{eV} .{ }^{36}$ The oxidation potentials for G15 and $\mathrm{R} 8$ are $+0.97 \mathrm{~V}$ and $+0.85 \mathrm{~V} \mathrm{v} \mathrm{Ag} / \mathrm{AgCl}$, respectively ${ }^{27}$; the optical absorption maximum gives a HOMO/LUMO gap of $575 \mathrm{~nm}(2.15 \mathrm{eV})$ and $660 \mathrm{~nm}(1.88 \mathrm{eV})$, respectively. The flat band potential of the ZnO ATRE crystal was estimated by MottSchottky measurements to be $-0.18 \mathrm{~V} \mathrm{v} \mathrm{Ag/AgCl}$. Assuming that upon aggregation any shift in the ground state of both dyes will be similar one can approximate the energy of the aggregate excited state. The aggregate optical absorption peaks are at $540 \mathrm{~nm}(2.30 \mathrm{eV})$ and $580 \mathrm{~nm}(2.13 \mathrm{eV})$ for G15 and R8 respectively and therefore $0.15 \mathrm{eV}$ and $0.25 \mathrm{eV}$ blue shifted from the monomer peaks. With regards to the effect of these differences in energetics on injection (or recombination), it is possible that the shift perturbs the relative injection efficiency of monomer compared to aggregate. However, in light of the similarities between the two dyes we do not consider this argument alone to be compelling.

One further difference between the work presented here and that of Rowley et al. is that in their study all ATR and photocurrent measurements were performed with excess dye present in the electrolyte solution (ethanol). Conversely, in this study, the cell was rinsed with solvent post sensitization followed by the introduction of 
hydroquinone and supporting electrolyte into the cell (acetonitrile based). Due to the significant differences in solvent polarity, it is expected that the surface morphology of the dye monomer/aggregates could be very different in these two solvents. Specifically, in the case of Rowley et al., it is probable that not all of the aggregates are fully in contact with the surface. On the contrary, in the case of a sensitized surface that has been rinsed and soaked in a non-solvent as in this work (acetonitrile), we speculate a more compact monolayer of aggregates that enhances the chance for injection into the so called "hot spots" for electron accepting and enhancing the efficiency of injection. Furthermore, R8 is significantly more prone to aggregation than G15. Indeed, the higher concentration of aggregates likely eventuate the observed phenomenon.

\section{Conclusion}

Simultaneous ATR and photocurrent measurements across a range of concentrations of sensitizing R8 dye demonstrates that monomer and aggregated species contribute approximately equally to the photocurrent on $\mathrm{ZnO}$ crystal surfaces across low concentration ranges. At higher dye concentrations (>10 uM), however, we observe a proportionally higher injection efficiency from aggregates at a $\mathrm{ZnO}$ single crystal surface. This is particularly interesting in comparison to the analogous work of Rowley et al., conducted using the 2,2' dimethylcarboxy-thiacarbocyanine iodide (G15, Fig. 1B) with a nearly identical molecular structure, except for a longer (by 2 carbon atoms) bridge relative to R8 (Figure 1). ${ }^{35}$

\section{Acknowledgement}

Thanks to J. G. Rowley for designing and troubleshooting the ATR flow cell and general expertise. We also thank Snowy Range Instruments for loan of the CCD detector, as well as TgK Scientific Ltd for assistance with SPECFIT analysis to deconvolute the monomer and dimer spectral shapes. We thank M. Spitler for insightful discussions. The authors wish to acknowledge the U.S. Department of Energy, Office of Science, Basic Energy Sciences, Division of Chemical Sciences, 
Geosciences and Biosciences for financial support through grant DE-FG0396ER14625.

\section{Supporting Information}

The Supporting Information is available free of charge on the ACS Publications website. The supporting information includes the APCE plots for data shown in Figure 4.

\section{References}

(1) O'Regan, B.; Grätzel, M. A Low-Cost, High-Efficiency Solar Cell Based on DyeSensitized Colloidal TiO2 Films. Nature 1991, 353 (6346), 737-740.

(2) Gratzel, M. Dye-Sensitized Solar Cells. J. Photochem. Photobiol. C-Photochem. Rev. 2003, 4, 145-153.

(3) Gratzel, M. Solar Energy Conversion by Dye-Sensitized Photovoltaic Cells. Inorg. Chem. 2005, 44, 6841-6851.

(4) Hardin, B. E.; Snaith, H. J.; McGehee, M. D. The Renaissance of Dye-Sensitized Solar Cells. Nat. Photonics 2012, 6, 162-169.

(5) Quintana, M.; Edvinsson, T.; Hagfeldt, A.; Boschloo, G. Comparison of DyeSensitized $\mathrm{ZnO}$ and TiO2 Solar Cells: Studies of Charge Transport and Carrier Lifetime. J. Phys. Chem. C 2007, 111 (2), 1035-1041.

(6) Keis, K.; Bauer, C.; Boschloo, G.; Hagfeldt, A.; Westermark, K.; Rensmo, H.; Siegbahn, H. Nanostructured ZnO Electrodes for Dye-Sensitized Solar Cell Applications. J. Photochem. Photobiol. Chem. 2002, 148 (1-3), 57-64.

(7) Zhang, Q.; Dandeneau, C. S.; Zhou, X.; Cao, G. ZnO Nanostructures for DyeSensitized Solar Cells. Adv. Mater. 2009, 21 (41), 4087-4108.

(8) Snaith, H. J.; Ducati, C. SnO2-Based Dye-Sensitized Hybrid Solar Cells Exhibiting Near Unity Absorbed Photon-to-Electron Conversion Efficiency. Nano Lett. 2010, 10 (4), 1259-1265.

(9) King, L. A.; Yang, Q.; Grossett, M. L.; Galazka, Z.; Uecker, R.; Parkinson, B. A. Photosensitization of Natural and Synthetic SnO2 Single Crystals with Dyes and Quantum Dots. J. Phys. Chem. C 2016.

(10) Nazeeruddin, M. K.; Kay, A.; Rodicio, I.; Humphry-Baker, R.; Mueller, E.; Liska, P.; Vlachopoulos, N.; Graetzel, M. Conversion of Light to Electricity by CisX2bis(2,2'-bipyridyl-4,4'-dicarboxylate)ruthenium(II) Charge-Transfer Sensitizers $(\mathrm{X}=\mathrm{Cl}-, \mathrm{Br}-, \mathrm{I}-, \mathrm{CN}-$, and $\mathrm{SCN}-)$ on Nanocrystalline Titanium Dioxide Electrodes. J. Am. Chem. Soc. 1993, 115 (14), 6382-6390.

(11) Shoute, L. C. T.; Loppnow, G. R. Excited-State Metal-to-Ligand Charge Transfer Dynamics of a Ruthenium(II) Dye in Solution and Adsorbed on TiO2 Nanoparticles from Resonance Raman Spectroscopy. J. Am. Chem. Soc. 2003, 125 (50), 15636-15646.

(12) Katoh, R.; Furube, A.; Yoshihara, T.; Hara, K.; Fujihashi, G.; Takano, S.; Murata, S.; Arakawa, H.; Tachiya, M. Efficiencies of Electron Injection from Excited N3 
Dye into Nanocrystalline Semiconductor $(\mathrm{ZrO} 2, \mathrm{TiO} 2, \mathrm{ZnO}, \mathrm{Nb} 2 \mathrm{O} 5, \mathrm{SnO} 2$, In2O3) Films. J. Phys. Chem. B 2004, 108 (15), 4818-4822.

(13) Doja, M. Q. The Cyanine Dyes. Chem. Rev. 1932, 11 (3), 273-321.

(14) Mishra, A.; Behera, R. K.; Behera, P. K.; Mishra, B. K.; Behera, G. B. Cyanines during the 1990s: A Review. Chem. Rev. 2000, 100, 1973-2011.

(13) Spectral Absorption and Fluorescence of Dyes in the Molecular State, Nature, 138, 1009-1010

(16) Planells, M.; Forneli, A.; Martínez-Ferrero, E.; Sánchez-Díaz, A.; Sarmentero, M. A.; Ballester, P.; Palomares, E.; O’Regan, B. C. The Effect of Molecular Aggregates over the Interfacial Charge Transfer Processes on Dye Sensitized Solar Cells. Appl. Phys. Lett. 2008, 92 (15), 153506.

(17) Mulhern, K. R.; Detty, M. R.; Watson, D. F. Aggregation-Induced Increase of the Quantum Yield of Electron Injection from Chalcogenorhodamine Dyes to TiO2. J. Phys. Chem. C 2011, 115 (13), 6010-6018.

(18) Mulhern, K. R.; Detty, M. R.; Watson, D. F. Effects of Surface-Anchoring Mode and Aggregation State on Electron Injection from Chalcogenorhodamine Dyes to Titanium Dioxide. J. Photochem. Photobiol. Chem. 2013, 264, 18-25.

(19) Eisfeld, A.; Briggs, J. S. The J- and H-Bands of Organic Dye Aggregates. Chem. Phys. 2006, 324 (2-3), 376-384.

(20) Gerischer, H.; Tributsch, H. Elektrochemische Untersuchungen Zur Spektralen Sensibilisierung von ZnO-Einkristallen. Berichte Bunsenges. Für Phys. Chem. 1968, 72 (3), 437-445.

(21) Tributsch, H.; Gerischer, H. Elektrochemische Untersuchungen Über Den Mechanismus Der Sensibilisierung Und Übersensibilisierung an ZnOEinkristallen. Berichte Bunsenges. Für Phys. Chem. 1969, 73 (3), 251-260.

(22) Memming, R.; Tributsch, H. Electrochemical Investigations on the Spectral Sensitization of Gallium Phosphide Electrodes. J. Phys. Chem. 1971, 75 (4), 562570.

(23) Tributsch, H.; Calvin, M. Electrochemistry of Excited Molecules: PhotoElectrochemical Reactions of Chlorophylls*. Photochem. Photobiol. 1971, 14 (2), 95-112.

(24) O’Regan, B.; Moser, J.; Anderson, M.; Graetzel, M. Vectorial Electron Injection into Transparent Semiconductor Membranes and Electric Field Effects on the Dynamics of Light-Induced Charge Separation. J. Phys. Chem. 1990, 94 (24), 8720-8726.

(22) O Reagan, B.; Gratzel, M. A low-cost, high-efficiency solar-cell based on dyesensitized colloidal $\mathrm{TiO}_{2}$ films. Nature 1991, 353, 737-740.

(26) Spitler, M. T.; Parkinson, B. A. Dye Sensitization of Single Crystal Semiconductor Electrodes. Acc. Chem. Res. 2009, 42 (12), 2017-2029.

(27) Watkins, K. J.; Parkinson, B. A.; Spitler, M. T. Physical Models for Charge Transfer at Single Crystal Oxide Semiconductor Surfaces as Revealed by the Doping Density Dependence of the Collection Efficiency of Dye Sensitized Photocurrents. J. Phys. Chem. B 2015.

(28) Ushiroda, S.; Ruzycki, N.; Lu, Y.; Spitler, M. T.; Parkinson, B. A. Dye Sensitization of the Anatase (101) Crystal Surface by a Series of Dicarboxylated Thiacyanine Dyes. J. Am. Chem. Soc. 2005, 127, 5158-5168. 
(29) Lu, Y.; Choi, D.; Nelson, J.; Yang, O.-B.; Parkinson, B. A. Adsorption, Desorption, and Sensitization of Low-Index Anatase and Rutile Surfaces by the Ruthenium Complex Dye N3. J. Electrochem. Soc. 2006, 153 (8), E131-E137.

(30) Choi, D.; Rowley, J. G.; Spitler, M.; Parkinson, B. A. Dye Sensitization of Four Low Index TiO2 Single Crystal Photoelectrodes with a Series of Dicarboxylated Cyanine Dyes. Langmuir 2013, 29 (30), 9410-9419.

(31) Lu, Y.; Spitler, M. T.; Parkinson, B. A. Photochronocoulometric Measurement of the Coverage of Surface-Bound Dyes on Titanium Dioxide Crystal Surfaces. $J$. Phys. Chem. B 2006, 110 (50), 25273-25278.

(32) Spitler, M. Lubke, M. and Gerischer, H. Studies of Dye Photooxidation at Semiconductor Electrodes Using Attenuated Total Reflection Techniques. Ber Bunsenges Phys Chem 1979, 83, 663-666.

(33) Kavassalis, C.; Spitler, M. T. Photooxidation of Thiacyanine Dyes at Zinc Oxide Single-Crystal Electrodes. J. Phys. Chem. 1983, 87 (16), 3166-3171.

(34) Natoli, L. M.; Ryan, M. A.; Spitler, M. T. J Aggregate Sensitization of Zinc Oxide Electrodes as Studied by Internal Reflection Spectroscopy. J. Phys. Chem. 1985, 89 (8), 1448-1453.

(35) Rowley, J. G.; Parkinson, B. A. Simultaneous Measurement of Absorbance and Quantum Yields for Photocurrent Generation at Dye-Sensitized Single-Crystal ZnO Electrodes. Langmuir 2013, 29 (45), 13790-13796.

(36) Kavassalis, C.; Spitler, M. T. Photooxidation of Thiacyanine Dyes at Zinc Oxide Single-Crystal Electrodes. J. Phys. Chem. 1983, 87 (16), 3166-3171.

(37) Sonntag, L. P.; Spitler, M. T. Examination of the Energetic Threshold for DyeSensitized Photocurrent at Strontium Titanate (SrTiO3) Electrodes. J. Phys. Chem. 1985, 89 (8), 1453-1457.

(38) Ehret, A.; Stuhl, L.; Spitler, M. T. Variation of Carboxylate-Functionalized Cyanine Dyes to Produce Efficient Spectral Sensitization of Nanocrystalline Solar Cells. Electrochimica Acta 2000, 45, 4553-4557.

(39) Armitage, B.; Retterer, J.; O’Brien, D. F. Dimerization of Cyanine Dyes in Water Driven by Association with Hydrophobic Borate Anions. J. Am. Chem. Soc. 1993, 115 (23), 10786-10790.

(40) Lenhard, J. R.; Hein, B. R. Effects of J-Aggregation on the Redox Levels of a Cyanine Dye. J. Phys. Chem. 1996, 100 (43), 17287-17296.

(41) Matsuzaki, H.; Murakami, T. N.; Masaki, N.; Furube, A.; Kimura, M.; Mori, S. Dye Aggregation Effect on Interfacial Electron-Transfer Dynamics in Zinc Phthalocyanine-Sensitized Solar Cells. J. Phys. Chem. C 2014, 118 (31), 1720517212.

(42) de Miguel, G.; Marchena, M.; Ziółek, M.; Pandey, S. S.; Hayase, S.; Douhal, A. Femto- to Millisecond Photophysical Characterization of Indole-Based Squaraines Adsorbed on TiO2 Nanoparticle Thin Films. J. Phys. Chem. C 2012, 116 (22), 12137-12148.

(43) Tatay, S.; Haque, S. A.; O’Regan, B.; Durrant, J. R.; Verhees, W. J. H.; Kroon, J. M.; Vidal-Ferran, A.; Gaviña, P.; Palomares, E. Kinetic Competition in Liquid Electrolyte and Solid-State Cyanine Dye Sensitized Solar Cells. J. Mater. Chem. 2007, 17 (29), 3037-3044. 
(44) Tachikawa, T.; Cui, S.-C.; Tojo, S.; Fujitsuka, M.; Majima, T. Nanoscopic Heterogeneities in Adsorption and Electron Transfer Processes of Perylene Diimide Dye on TiO2 Nanoparticles Studied by Single-Molecule Fluorescence Spectroscopy. Chem. Phys. Lett. 2007, 443 (4-6), 313-318. 